\title{
A FERROFLUID-BASED PRESSURE SENSOR FOR BIOMEDICAL APPLICATIONS
}

\author{
Girish Chitnis ${ }^{* 1,3}$ and Babak Ziaie ${ }^{2,3}$
}

\author{
${ }^{1}$ School of Mechanical Engineering, Purdue University, West Lafayette, Indiana, USA \\ ${ }^{2}$ School of Electrical and Computer Engineering, Purdue University, West Lafayette, Indiana, USA \\ ${ }^{3}$ Birck Nanotechnology Center, Purdue University, USA
}

\begin{abstract}
This paper presents a new pressure sensor design based on magnetic liquid displacement and its corresponding inductance change. The design of pressure sensor is presented followed by its fabrication and in vitro test results. Experimental results show a nonlinear relationship between applied pressure and change in resonant frequency. The initial linear region $(0-20 \mathrm{mmHg})$ shows a sensitivity of $6 \mathrm{kHz} / \mathrm{mmHg}$. Simple design and easy fabrication process make this pressure sensor an attractive option for many biomedical applications.
\end{abstract}

\section{INTRODUCTION}

Wireless measurement of physiological pressures has been pursued for several decades following the pioneering work of Farrar et al. in 1957 to measure gastrointestinal pressure [1]. A decade later, further miniaturization of implantable pressure sensors was utilized by Collins [2] to measure intraocular pressure using a passive LC transponder. Since the rise of MEMS and microfabrication technology over the past couple of decades, many pressure sensors have been reported that are designed to measure pressure at various anatomical locations such as blood vessels [3], [4], eye [5-8], bladder [9], cranium [10], etc. Efforts in this area have been focused on passive (LC) and active (pressure sensor plus interface and RF electronics, powered through inductive methods or batteries) transponders. Passive devices have an inherent advantage over active ones since they do not need any external power source. Most passive transponders rely on changes in capacitance or inductance for pressure measurement. Although conceptually simple, their fabrication requires complex techniques. Here we present a much simpler passive pressure sensing transponder based on the novel concept of ferrofluid movement in and around an integrated coil. Inductance of the coil varies with applied pressure due to transfer of ferrofluid trapped in the sensor. Since the coil itself has some built-in stray capacitances, no external capacitor is needed to create the LC tank (inductor selfresonant frequency is therefore a function of applied pressure). Although there have been reports on ferrofluid-based gas flow sensor [11], inclinometer [12], and LVDT sensor [13]; the transducer presented here is the first implementation of a wireless passive pressure sensor using ferrofluid.

\section{DESIGN}

The working mechanism of the ferrofluid-based pressure sensor relies on high magnetic permeability of dispersed ferrite nanoparticles and fluidity of the dispersion medium. Figure 1 depicts the concept of the ferrofluid-based pressure sensor. It consists of four major components: 1) a needle, 2) ferrofluid, 3) a planar coil, and 4) a polymeric membrane. The needle penetrates a body cavity by piercing through a membrane such as sclera, blood vessel wall, or bladder wall to establish a direct hydraulic contact between ferrofluid and the biological inner fluid (aqueous humor, blood, urine, etc.) (Fig.1a). When internal pressure is higher than the atmospheric pressure, ferrofluid flows through the needle and the hole at the center of the coil, deflecting the polymeric (a)

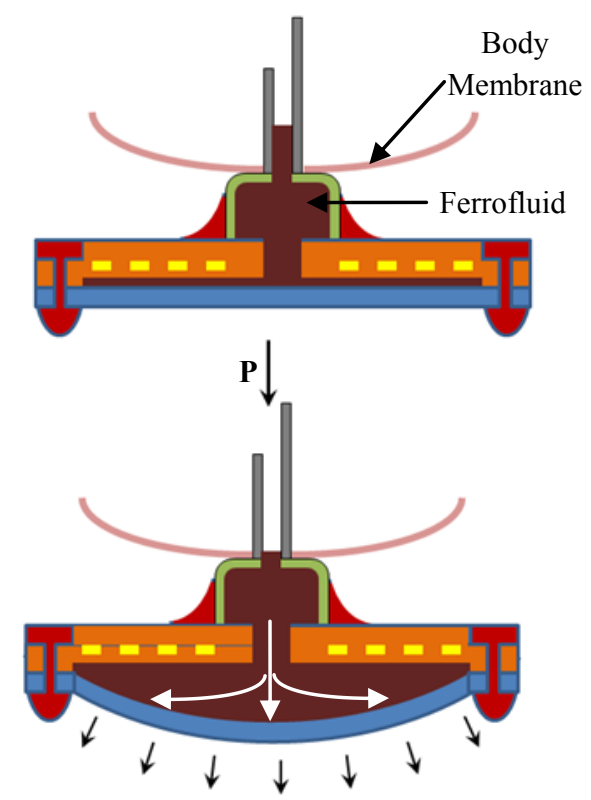

Figure 1: Schematic diagram of ferrofluid pressure sensor (dimensions not to scale)

membrane away from the coil (Fig. 1b). As a result, ferrofluid occupies more volume on top of the coil. Since inductance is directly proportional to the volume of the magnetic material in proximity to the coil, the pressure increase leads to an increase in the inductance (assuming the density of nanoparticles in the ferrofluid is constant). In our structure, stray capacitance is fixed and is not affected by change in the pressure; leading to a decrease in the self-resonant frequency of the coil as external pressure increases. For a given pressure, the change in resonant frequency of the coil depends on deflection of the polymeric membrane as well as magnetic permeability of ferrofluid. The geometry (radius and thickness) and material of the membrane can be altered to achieve desired sensitivity and range. Magnetic permeability of the ferrofluid can be altered by changing concentration of ferrite nanoparticle in the colloid. In the present design, we use $100 \mathrm{~m}$ thick Polyethylene terephthalate (PET) sheet with deflectable radius of $10 \mathrm{~mm}$ as polymeric membrane. The ferrofluid (APG 311 by Ferrotec, Inc.) has ferrite volume concentration of $2 \%$ (particle size $10 \mathrm{~nm}$ ) dispersed in synthetic hydrocarbon with overall magnetic permeability of 1.1 (measured at $\mathrm{f}=10 \mathrm{MHz}$ ). A polyimide based spiral coil with 20 turns, $18 \mathrm{~m}$ thick copper (2 layers) and $25 \mathrm{~m}$ polyimide (3 layers) is used for its high quality factor.

\section{FABRICATION}

Figure 2 shows the fabrication process flow of the ferrofluidbased pressure sensor. Polyimide flexcoil $(L \approx 10 \quad H)$ was used for fabrication of this prototype (Fig. 2a). The coil was designed in- 
(a)

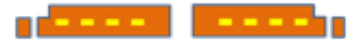

(c)

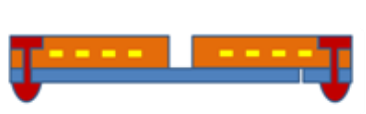

(e)

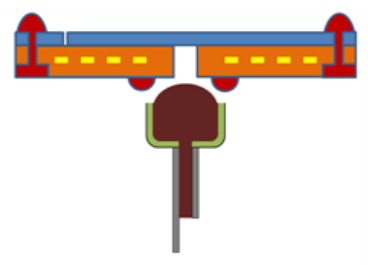

(g)

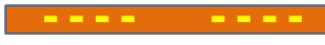

(b)

(d)

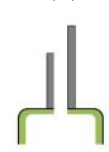

(f)

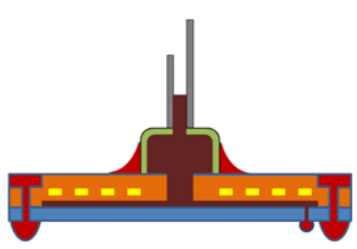

(h)
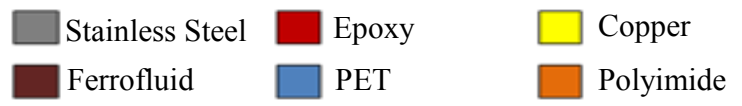

Figure 2: Fabrication process flow.

house but fabrication was outsourced to Parlex USA, Inc. A 100 $m$ thick PET sheet (transparency) was used as the polymeric membrane (Fig. 2b). Both flex-coil and transparency were machined to desired shape using a $\mathrm{CO}_{2}$ laser (Universal® Laser Systems), (Fig. 2c-d). The coil and membrane were bonded together using standard five minute epoxy (Fig. 2e). High viscosity epoxy was used to ensure that the membrane only bonds at the edges. Holes were laser-drilled near the edges to ensure a leakproof anchor. Note that an additional space around the coil needs to be provided for the anchors which lead to the physical size of the coil being different from its electrical size. Similarly, movable diameter of the PET membrane is smaller than its actual physical size. Commercially available hypodermic needle (26 gauge) was

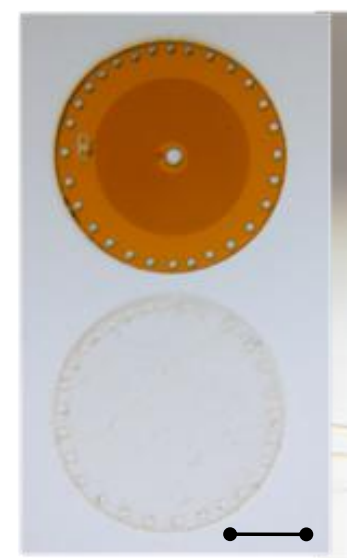

(a)

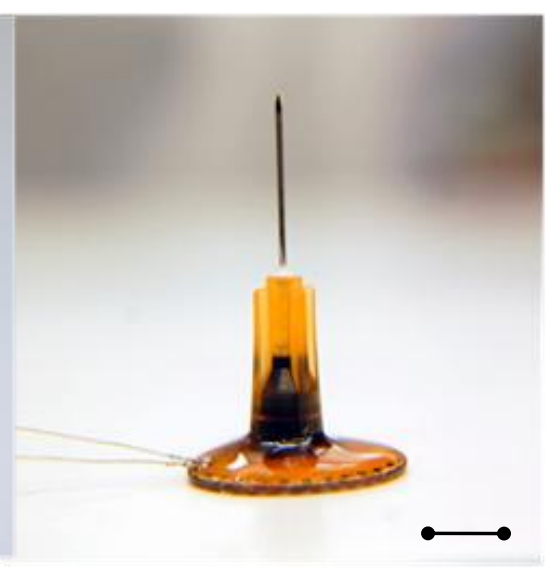

(b)
Figure 3: (a) Laser machined flex-coil and transparency (b) assembled ferrofluid pressure sensor pressure sensor (Scale bar: $5 \mathrm{~mm}$ ). mechanically machined to create the ferrofluid chamber (Fig. 2f). Chamber and needle prefilled with ferrofluid, was then bonded to the coil/membrane assembly using epoxy glue (Fig. 2 g). An escape hole was provided in the transparency to allow air to purge and allow the ferrofluid to completely fill the gaps. This escape hole was sealed later using epoxy glue (Fig. 2h). Figure 3 shows photographs of the laser-machined coil and transparency (Fig. 3a) along with a completely assembled ferrofluid based pressure sensor (Fig. 3b). Table 1 lists all the final parameters of the pressure sensor.

Table 1: Ferrofluid pressure sensor parameters

\begin{tabular}{lc}
\hline Polymeric membrane \\
\hline Thickness (mm) & 0.100 \\
Diameter & 10 \\
Movable (mm) & 15 \\
Total (mm) & \\
Coil & 0.250 \\
Thickness (mm) & 10 \\
Diameter & 15 \\
Electrical (mm) & \\
Physical (mm) & Gauge 26 \\
Hypodermic Needle & 0.260 \\
I.D. $($ mm) & 0.464 \\
O.D. $(\mathrm{mm})$ & 10 \\
Length (mm) &
\end{tabular}

\section{RESULTS AND DISCUSSION}

The pressure sensor was characterized against a water column pressure as shown in Figure 4a. The needle was inserted in a water-filled rubber balloon to mimic its insertion through a body cavity (Fig. 4b). Self-resonant frequency of the sensor was measured using an impedance analyzer. It is important to note that although here the frequency is measured through direct wired connection to the analyzer, similar measurement can be performed wirelessly by using phase-dip or other techniques. Resonant frequency was measured for pressures from 0 to $50 \mathrm{mmHg}$ while increasing and decreasing the pressure in steps of $5 \mathrm{mmHg}$. Figure 5a shows the measured impedance of the device at various pressures (not all the measurements are shown to maintain clarity of the diagram). The resonant frequency plotted against applied pressure is shown in Figure 5b.

It is safe to assume that if the nanoparticle concentration is kept constant, the change in inductance is proportional to volume of the ferrofluid surrounding the coil. Considering that the circular membrane would deflect to a spherical segment, change in ferrofluid volume will be proportional to the cube of deflection. Hence if we assume linear deflection region for the polymer membrane, change in inductance is expected to be proportional to the cube of applied pressure. As expected, figure 5b shows a very good fit for such cubic trend. One can consider the response to be almost linear in the range $(0-20 \mathrm{mmHg})$ where it shows a sensitivity of $\sim 6 \mathrm{kHz} / \mathrm{mmHg}$. Sensitivity and linear range of the sensor can be easily improved by simply adjusting geometry of the deflecting membrane. Considering the current thickness of $100 \mu \mathrm{m}$ there is considerable room to improve sensitivity. 


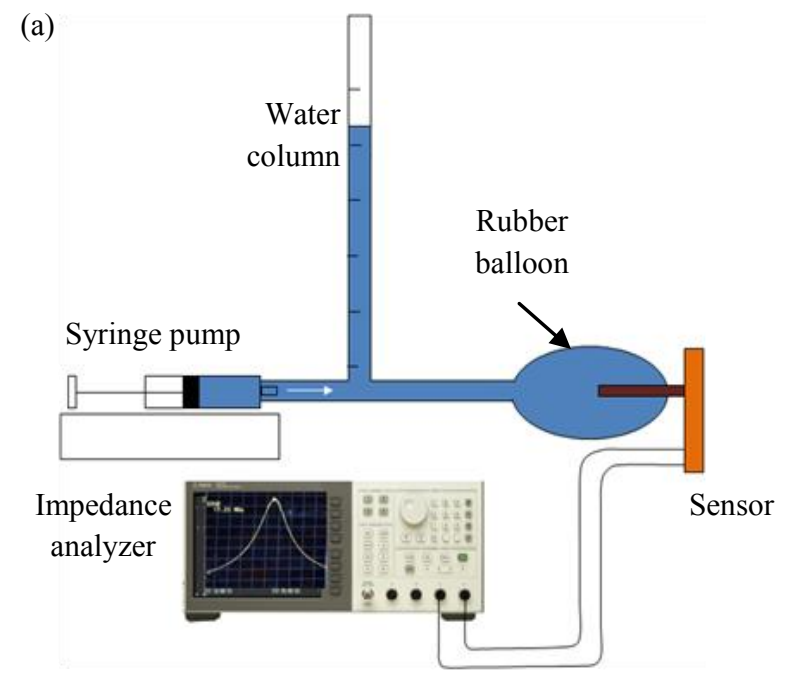

(b)

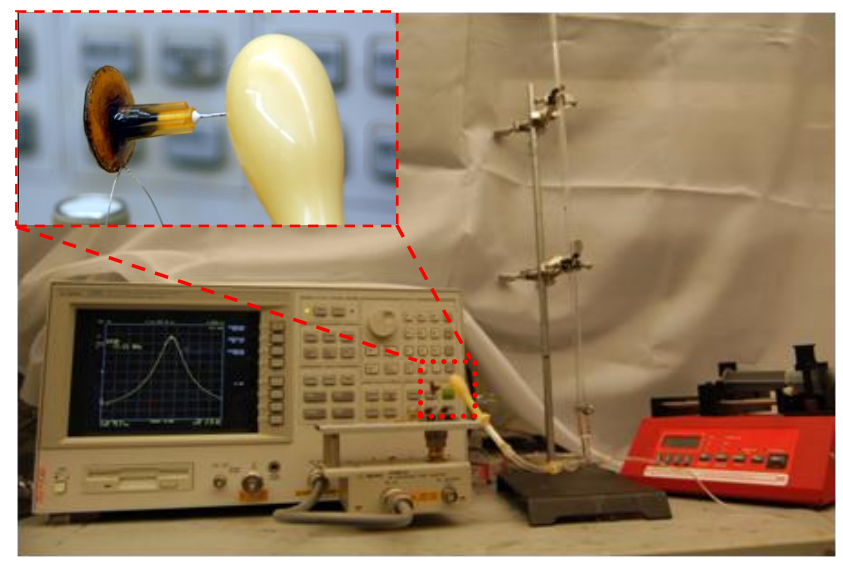

Figure 4: (a) Schematic diagram of the experimental set up (b) Photograph of the experimental set up (inset shows pressure sensor inserted in a rubber balloon)

It is important to note that the sensor uses only one coil in open configuration. Since there is no need to bring the contact out one can fabricate such coil in a single layer of metallization. Also, since this sensor does not need an additional capacitor, any established coil fabrication technique can be used to achieve low resistance and high quality factor.

Apart from the advantages, there are some concerns that need to be addressed. In the current design ferrofluid is directly exposed to exteriors through needle opening. This can lead to leakage of ferrofluid leading to a dysfunctional sensor. Further if the ferrofluid level drops below the needle while pressurizing, it is easy to trap a bubble inside the chamber which would not only obstruct ferrofluid movement but also affect effective magnetic permeability of the system. Use of a high gauge (i.e. small inner diameter) needle resolves these issues to some extent since surface tension helps confining the ferrofluid. However a physical barrier separating the exterior from ferrofluid can improve reliability significantly. Although biocompatibility of the device in its current form is not validated, most of the structural components can be replaced if needed. The only irreplaceable component, ferrite nanoparticles, is shown to be biocompatible [14, 15], and it has

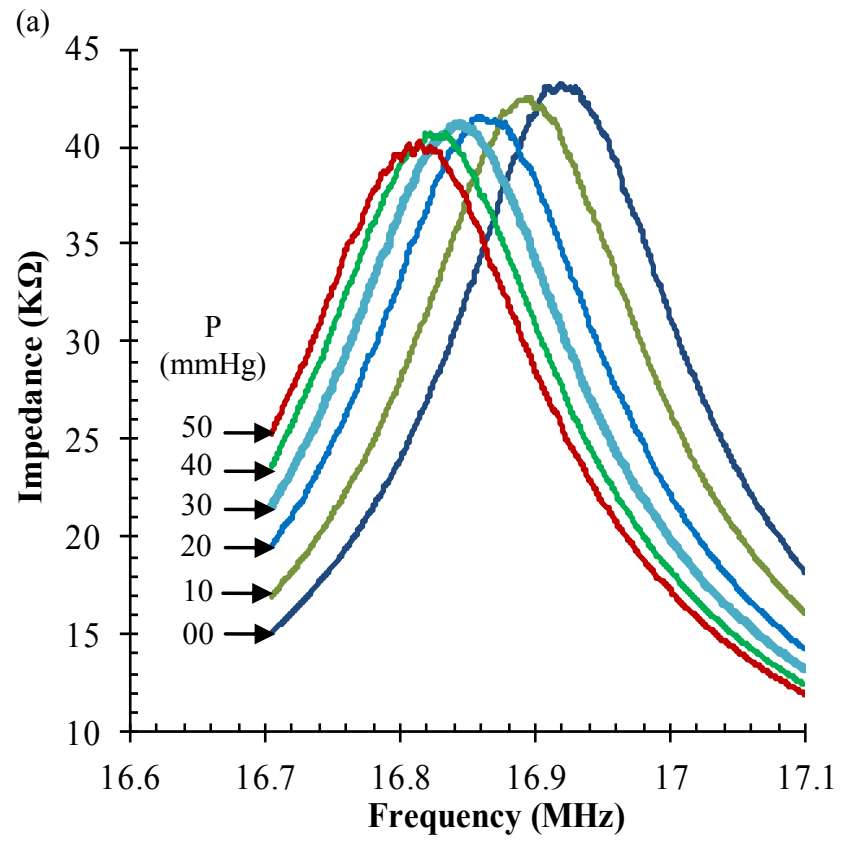

(b)

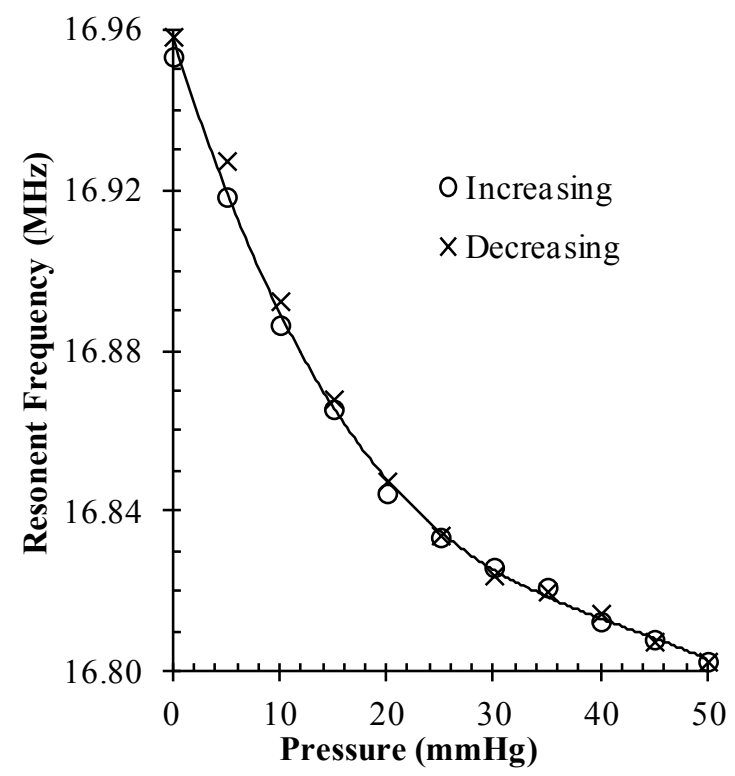

Figure 5: Measurements (a) Frequency sweep of impedance measurement at various applied pressures (b) Self-resonant frequency $(\mathrm{MHz})$ plotted against applied pressure $(\mathrm{mmHg})$

been proposed as an implant for the treatment of retinal detachment [16]. Finally, for some implant sites such as urinary bladder, which is not exposed to atmospheric air, an absolute pressure sensor is preferable. Ferrofluid pressure sensor can be easily converted to absolute pressure sensor by simply including a reference chamber across the polymeric membrane as shown in Fig. 6.

\section{CONCLUSION}

In this paper, we discussed a new pressure sensing technique based on magnetic liquid displacement and corresponding 


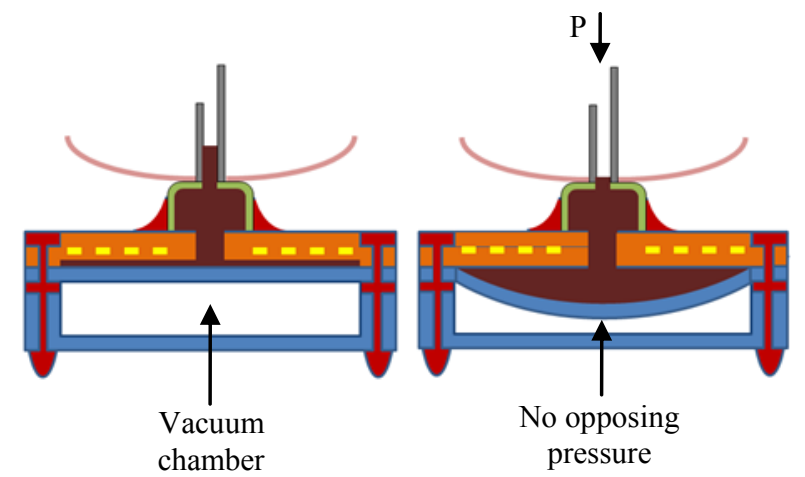

Figure 6: Proposed schematic diagram of an absolute pressure sensor based on ferrofluid movement.

inductance change. A pressure sensor was designed, fabricated, and tested in vitro. Measurements show a cubic relation between applied pressure and inductance change. The initial linear region $(0-20 \mathrm{mmHg})$ shows a sensitivity of $6 \mathrm{kHz} / \mathrm{mmHg}$. Finally, a design variation was suggested to fabricate an absolute pressure sensor based on ferrofluid movement. Simple design and easy fabrication process make this pressure sensor an attractive option for many biomedical applications.

\section{ACKNOWLEDGEMENTS}

The authors wish to thank staff of the Birck Nanotechnology Center at Purdue University for their assistance in fabrication of the transponder

\section{REFERENCES}

[1] J. T. Farrar, V. K. Zworykin, and J. Baum, "PressureSensitive Telemetering Capsule for Study of Gastrointestinal Motility," Science, vol. 126, no. 3280, pp. 975-976, Nov. 1957.

[2] C. C. Collins, "Miniature passive pressure transensor for implanting in the eye," IEEE transactions on bio-medical engineering, vol. 14, no. 2, pp. 74-83, Apr. 1967.

[3] M. G. Allen, "Micromachined endovascularly-implantable wireless aneurysm pressure sensors: from concept to clinic," in The 13th International Conference on Solid-State Sensors, Actuators and Microsystems, 2005. Digest of Technical Papers. TRANSDUCERS '05, 2005, vol. 1, pp. 275-278.

[4] B. Ziaie and K. Najafi, "An Implantable Microsystem for Tonometric Blood Pressure Measurement," Biomedical Microdevices, vol. 3, no. 4, pp. 285-292, 2001.

[5] T. Eggers et al., "Wireless intra-ocular pressure monitoring system integrated into an artificial lens," in Microtechnologies in Medicine and Biology, 1st Annual International, Conference On. 2000, 2000, no. 1, pp. 466-469.

[6] K. Stangel, S. Kolnsberg, D. Hammerschmidt, B. Hosticka, H. Trieu, and W. Mokwa, "A programmable intraocular CMOS pressure sensor system implant," Solid-State Circuits, IEEE Journal of, vol. 36, no. 7, pp. 1094-1100, 2001.
[7] D. C. Rodger, S. Saati, and M. S. Humayun, "Microfabricated Implantable Parylene-Based Wireless Passive Intraocular Pressure Sensors," Journal of Microelectromechanical Systems, vol. 17, no. 6, pp. 1342-1351, Dec. 2008.

[8] G. D. Chitnis, T. Maleki, B. Samuels, L. B. Cantor, and B. Ziaie, "An ocular tack for minimally invasive continuous wireless monitoring of intraocular pressure," in 2012 IEEE 25th International Conference on Micro Electro Mechanical Systems (MEMS), 2012, no. February, pp. 922-925.

[9] J. Melgaard and N. J. M. Rijkhoff, "Detecting the onset of urinary bladder contractions using an implantable pressure sensor.," IEEE transactions on neural systems and rehabilitation engineering: a publication of the IEEE Engineering in Medicine and Biology Society, vol. 19, no. 6, pp. 700-8, Dec. 2011.

[10] M. Ghannad-Rezaie, L. J.-S. Yang, H. J. L. Garton, and N. Chronis, "A Near-Infrared Optomechanical Intracranial Pressure Microsensor," Journal of Microelectromechanical Systems, vol. 21, no. 1, pp. 23-33, Feb. 2012.

[11] H. Oka and J. Iwata, "Fundamental Behavior of Micro Gas Flow Sensor Using Magnetic Fluid," IEEE Translation Journal on Magnetics in Japan, vol. 2, no. 6, pp. 563-565, Jun. 1987.

[12] B. Ando, A. Ascia, and S. Baglio, "A Ferrofluidic Inclinometer in the Resonant Configuration," IEEE Transactions on Instrumentation and Measurement, vol. 59, no. 3, pp. 558-564, Mar. 2010.

[13] M. Felix, A. Lizarraga, A. Islas, and A. Gonzalez, "Analysis of a ferrofluid core LVDT displacement sensor," in IECON 2010 - 36th Annual Conference on IEEE Industrial Electronics Society, 2010, pp. 1769-1772.

[14] A. Tomitaka, A. Hirukawa, T. Yamada, S. Morishita, and Y. Takemura, "Biocompatibility of various ferrite nanoparticles evaluated by in vitro cytotoxicity assays using HeLa cells," Journal of Magnetism and Magnetic Materials, vol. 321, no. 10, pp. 1482-1484, May 2009.

[15] D. H. Kim, S.-H. Lee, K.-N. Kim, K.-M. Kim, I.-B. Shim, and Y.-K. Lee, "Cytotoxicity of ferrite particles by MTT and agar diffusion methods for hyperthermic application," Journal of Magnetism and Magnetic Materials, vol. 293, no. 1, pp. 287292, May 2005.

[16] O. T. Mefford et al., "Field-induced motion of ferrofluids through immiscible viscous media: Testbed for restorative treatment of retinal detachment," Journal of Magnetism and Magnetic Materials, vol. 311, no. 1, pp. 347-353, Apr. 2007.

\section{CONTACT}

*G. D. Chitnis, tel: +1-413-561-5226; gchitnis@purdue.edu 